\title{
Racial distribution of urology workforce in United States in comparison to general population
}

\author{
Samuel L. Washington III ${ }^{1}$, Nima Baradaran ${ }^{1}$, Thomas W. Gaither ${ }^{1}$, Mohannad A. Awad ${ }^{1}$, Gregory P. \\ Murphy $^{1}$, Tracy M. Downs ${ }^{2}$, Benjamin N. Breyer ${ }^{1,3}$ \\ ${ }^{1}$ Department of Urology, School of Medicine, University of California San Francisco, San Francisco, CA, USA; ${ }^{2}$ Department of Urology, School of \\ Medicine, University of Wisconsin, Madison, WI, USA; ${ }^{3}$ Department of Urology, Zuckerberg San Francisco General Hospital and Trauma Center, \\ San Francisco, CA, USA \\ Contributions: (I) Conception and design: SL Washington 3rd, TW Gaither, MA Awad, GP Murphy, BN Breyer; (II) Administrative support: BN \\ Breyer; (III) Provision of study materials or patients: BN Breyer; (IV) Collection and assembly of data: SL Washington 3rd, TW Gaither; (V) Data \\ analysis and interpretation: All authors; (VI) Manuscript writing: All authors; (VII) Final approval of manuscript: All authors. \\ Correspondence to: Benjamin N. Breyer, MD, MAS. Department of Urology, School of Medicine, University of California San Francisco, 400 \\ Parnassus Ave, UCSF Box 0738, San Francisco, CA 94143, USA . Email: benjamin.breyer@ucsf.edu.
}

Background: To compare the current racial/ethnic characteristics of practicing urologists to the U.S. population by American Urological Association (AUA) census geographic region.

Methods: We compared urologist demographics from the 2014 AUA census to U.S. census data. Underrepresented in medicine (URM) status was defined as African-American (AA) or Hispanic race/ethnicity. Percent differences by AUA section were calculated by subtracting weighted frequencies of race/ethnicity for urologists from the general population. A negative percent difference denotes underrepresentation of urologists relative to the general population; positive percent difference denotes overrepresentation.

Results: URM urologists $(\mathrm{n}=728,6.5 \%)$ were younger and more often female than non-URM counterparts. Overall, AA and Hispanic urologists were underrepresented in most sections while Caucasian and Asian urologists were overrepresented. AA urologists were most underrepresented in the East SouthCentral section $(-34.4 \%)$. Hispanic urologists (-38\%) were most underrepresented in the Pacific section (-38\%). Overall, the percentage of URM urologists, compared to non-URM urologists, were highest in the South Atlantic [37.9\% (276/728) vs. 19.2\% (1,984/10,319), $\mathrm{P}<0.01]$ and West South-Central [15.9\% (116/728) vs. $11.1 \%(1,143 / 10,319), \mathrm{P}<0.01]$.

Conclusions: URM urologists tend to be younger with a higher proportion of female providers, indicating a shift in race and gender. URM urologists were most underrepresented in the East South-Central and Pacific sections.

Keywords: Diversity; urology; under-representation

Submitted Mar 21, 2018. Accepted for publication May 14, 2018.

doi: 10.21037/tau.2018.05.16

View this article at: http://dx.doi.org/10.21037/tau.2018.05.16

\section{Introduction}

The patient-physician relationship is built on trust and communication. Quality of care, including patient satisfaction (1,2) and follow-through on treatment (3-5) is directly impacted by the patient-physician relationship. Concordance is defined as a similarity or shared identity.
In the patient-physician relationship, concordance may be based on race, sex, or age (5). There is a growing body of evidence in health disparity research that racial concordance between patient and physician can contribute to patient's trust, satisfaction, resource utilization, and decision-making participation (6-10). However, data is controversial at times as other investigators have found no association between 
age or race concordance and variety of health indices such as pain, and patient-perception of high-quality care (11-13).

Street $e t$ al. have demonstrated that perceived personal similarity is associated with higher ratings of trust, satisfaction, and intention to adhere. In addition, race concordance is the primary predictor of perceived ethnic similarity (5). The majority of research on racial concordance has been in primary care $(3,14)$, pediatrics $(11)$, geriatrics, and oncology (15). To date the racial concordance of urologic workforce in the United States has not been studied.

The first step in understanding the impact of racial congruence on health metrics in urology is to evaluate the racial distribution of physicians with regards to the patients they serve. We aim to characterize the racial/ethnic representation of the urologic workforce and compare it with their respective American Urologic Association (AUA) section-level race-specific population in order to identify underrepresentation in the field as a surrogate for concordance.

\section{Methods}

We obtained de-identified unweighted county-level population estimates from the Area Health Resources Files system, extrapolated from the 2010 U.S. Census. Counts were categorized by self-reported race: White, Black/ African American (AA), Hispanic and Asian. White/nonHispanic was categorized as White and Black/non-Hispanic categorized as AA to avoid duplication of Hispanic patients who also self-reported as Black or White. Aggregate population counts by state were used to generate state-level race-specific population estimates. Underrepresentation in medicine (URM) individuals were defined as those selfreported as AA or Hispanic; non-URM individuals were those self-reported as White or Asian.

Urologist demographic information was obtained from the 2014 AUA Census. The dataset used post-stratification weighting to generate census samples of the urologist population. The cohort was then stratified by AUA geographic census section. URM providers were those who self-reported as AA or Hispanic; non-URM patients were those self-reported as White or Asian. White/nonHispanic subjects were categorized as White and Black/ non-Hispanic urologist subjects as AA to avoid duplicate counting. An estimated 11,703 urologists were extrapolated from the 2014 AUA census. A total of $4.83 \%$ were not used due to missing race/ethnicity data. For each dataset, those of mixed heritage, "other" or unknown were not reported due to inability to make comparisons for this analysis. Similarly, counts of these other groups were not included in the total population calculations to provide more accurate estimates of the true population composition. As a result, totals displayed may not equal $100 \%$ due to the proportions for these groups not being reported.

\section{Statistical analysis}

Summary statistics stratified by URM status were utilized to compare the urologic and general populations. Frequency tables were used to describe categorical variables and medians with interquartile ranges (IQR) for nonparametric continuous variables. A Chi squared test was used to compare categorical variables. Mann-Whitney test was used to compare medians and distributions of nonparametric continuous variables.

The AUA census data and U.S. census, by AUA census region, were then compared to assess demographic differences between the populations. U.S. census state-level data was aggregated based upon the state groups within each AUA geographic section. Within each AUA section, percentage composition of the general population by race and URM status was calculated by dividing the race-specific total by the population total. This was repeated using the urologist population.

Percent difference was calculated by subtracting each section's race-specific proportion of the general population from the corresponding AUA race-specific proportion. Given there are no established cutoffs for degree of representation, those with differences less than $5 \%$ were considered well-represented. Those with larger positive differences were over-represented while those with larger negative differences were under-represented. We based our comparative statistical analyses on the assumption that temporal changes in the total U.S. population and the racial/ethnic composition of both the national and urologic workforce populations were small and insignificant. Statistical analyses were performed using STATA/IC 14.2 (StataCorp, College Station, TX, USA). A P value $<0.05$ was considered statistically significant.

\section{Results}

\section{Overall workforce}

A total of 11,138 practicing urologists were included. A total of 728 (6.5\%) urologists were categorized as URM (262 
Table 1 Demographic data of urologists, stratified by URM status

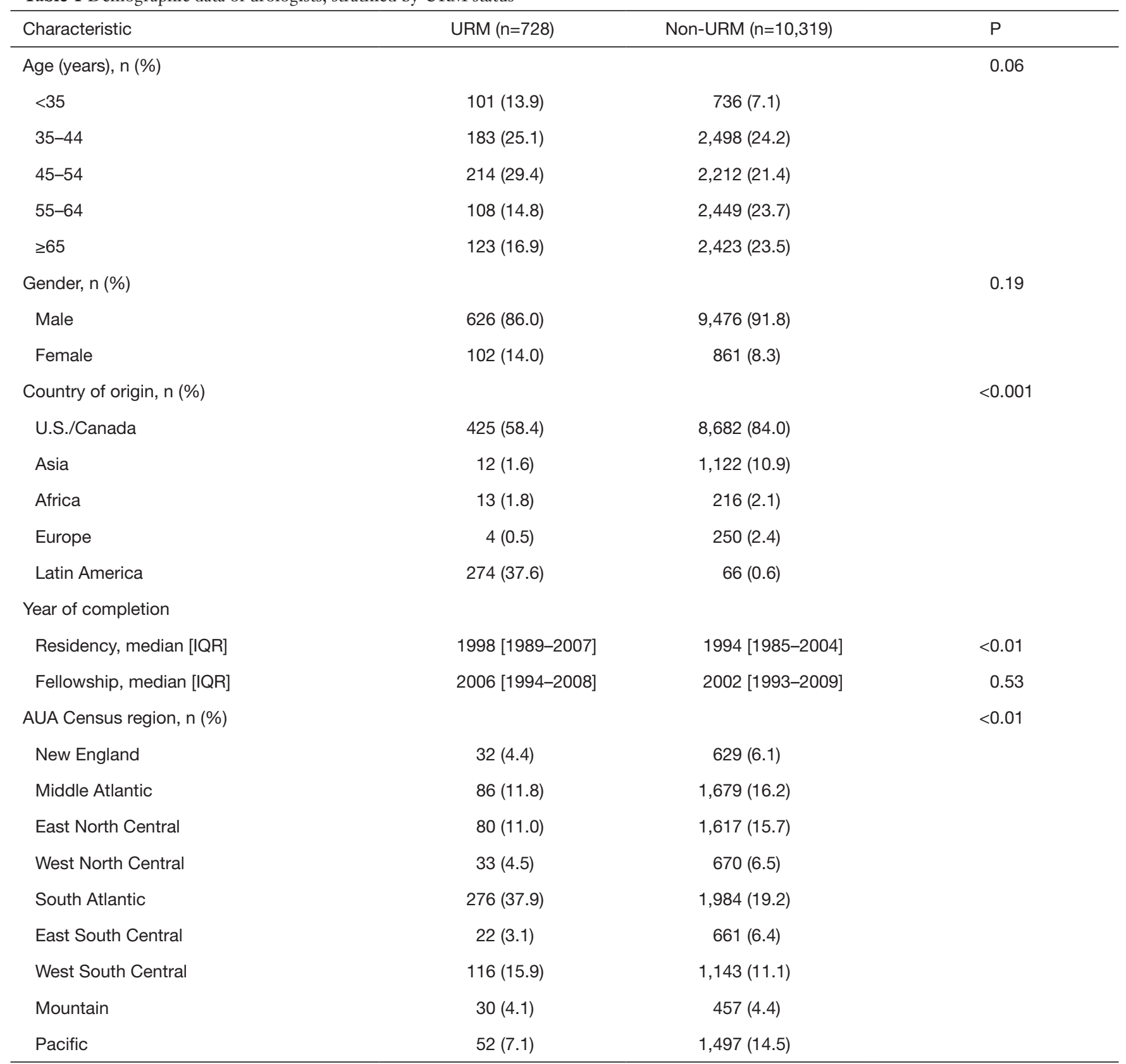

Weighted counts used for each estimate, rounded to nearest whole number (missing data are not shown but included in the total counts). URM, underrepresented in medicine; AUA, American Urological Association.

AA and 466 Hispanic). Table 1 displays demographic data of practicing urologists by URM status. URM urologists were more often younger [68.4\% (498/728) of URM providers under 55 years old URM vs. $52.8 \%(5,446 / 10,319)$ nonURM providers under 55 years, $\mathrm{P}=0.06]$ and female $[14.0 \%$ $(102 / 728)$ vs. $8.3 \%(861 / 10,319), \mathrm{P}=0.19]$. There appeared to be a trend towards URM urologists being younger than their non-URM colleagues though not statistically significant. The proportion of women within the urologist workforce increased within younger age groups $(\mathrm{P}<0.001)$. URM providers finished residency later than non-URM providers (median year 1998 vs. 1994, $\mathrm{P}<0.01$ ). The majority 
Table 2 Training and occupational data on AUA urologists, stratified by URM status

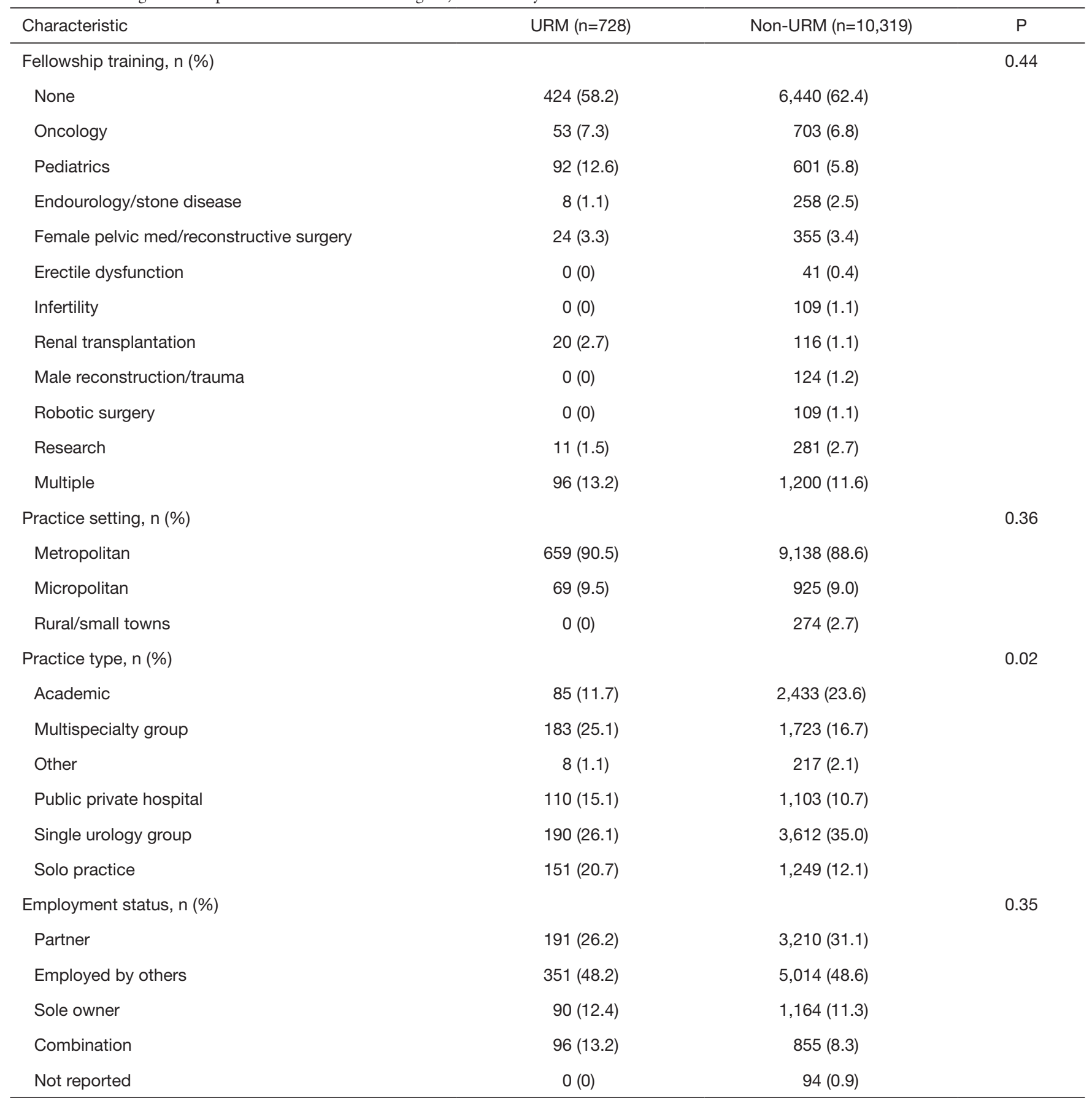

Estimates reported are weighted and rounded to the nearest whole number (missing data are not shown but included in the total counts). URM, underrepresented in medicine; AUA, American Urological Association.

in both groups did not pursue fellowship training (Table 2). Year of fellowship completion did not differ significantly between the groups. Pediatric urology was most common fellowship reported amongst URM providers while
Urologic Oncology was most common amongst non-URM urologist. Practicing URM urologists were concentrated in specific geographic regions relative to their non-URM colleagues [37.9\% (276/728) vs. 19.2\% (1,984/10,319) 
in South Atlantic and 15.9\% (116/728) vs. 11.1\% $(1,143 / 10,319)$ in West South Central, $\mathrm{P}<0.01]$. More often, URM urologists worked in multispecialty [25.1\% (183/728) vs. $16.7 \%(1,723 / 10,319)]$ or solo practices [20.7\% (151/728) vs. $12.1 \%(1,249 / 10,319)], \mathrm{P}=0.02$.

\section{Academics workforce}

Academic urologists comprised less than one-fourth of the urologic workforce $(2,518 / 11,138,22.6 \%)$ with $11.7 \%$ $(85 / 728)$ of URM providers and $23.6 \%(2,433 / 10,319)$ of non-URM providers reporting working in an academic setting $(\mathrm{P}=0.02)$. Table 3 shows demographic characteristics of academic urologists by URM status. The academic workforce mirrored the non-academic urologist population with increasing proportions of female urologists in the younger subgroups. URM academic urologists were typically younger than their non-URM colleagues [proportion greater than 55 years old, $8.2 \%(7 / 85) v s$. $33.9 \%(824 / 2,433), \mathrm{P}=0.29]$. URM academics were more often originated from countries other than the U.S. and Canada [30.6\% (26/85) vs. $19.6 \%(478 / 2,433), \mathrm{P}<0.001]$ compared to non-URM academics. Latin America (22/85, $25.9 \%)$ and Africa (4/85, 4.7\%) were most commonly reported for foreign-born academic URM urologists. Academic urologists did not differ significantly in the year of completing residency, fellowship, nor AUA census region. Academic URM urologists were most often employees within the practice $(73 / 85,85.9 \%)$ whereas academic nonURM urologists more often reported having partner status [7.4\% (181/2,433) vs. $4.7 \%$ (4/85), $\mathrm{P}=0.01]$.

\section{Comparison to general population}

URM patients were least common in the East SouthCentral section (18.7\%) and most in the Mountain and Pacific sections (47.4\% and $36.2 \%$, respectively). Proportions of non-URM patients were highest in the New England section (83.5\%) and lowest in the Mountain sections $(50.9 \%)$. Within the urologist workforce, URM urologists were most common in the South Atlantic and West South-Central regions $(37.9 \%$ and $16.0 \%$ respectively). Non-URM providers were most common in the South Atlantic and Middle Atlantic regions (19.2\% and $16.2 \%)$.

By comparing racial/ethnic distributions between the general population and urologist population, we quantified differences in representation (Figure 1). AA and Hispanic populations were underrepresented in all sections. The opposite was generally seen for Caucasian and Asian populations. Percent differences by AUA section ranged from $-0.4 \%$ to $+39.8 \%$ for the White population, $-15.9 \%$ to $-4.5 \%$ for the AA population, $-33.5 \%$ to $-3.2 \%$ for the Hispanic population and $-1.0 \%$ to $15.0 \%$ for the Asian population. The Caucasian population was most overrepresented in the Mountain section $(+39.8 \%)$ and well-represented in the New England section (-0.4\%). The AA population was most underrepresented in the East North-Central section (-15.9\%); least in the New England section $(-6.80 \%)$. The Hispanic population was most underrepresented in the Mountain section $(-33.5 \%)$ and well-represented in the New England section (-3.2\%). The Asian population was most overrepresented in the Pacific section $(+15.0 \%)$ and well-represented in the New England section (-1.0\%). URM groups were underrepresented in all sections; the Mountain section with the largest percentage difference $(-43.7 \%)$ and the New England section with the least $(-12.7 \%)$. The non-URM population was most overrepresented in the Mountain section (+36.3\%) and least in the New England section (+8.2\%).

\section{Discussion}

The findings of this study are the initial step to evaluate racial/ethnic concordance between urologists and their respective patients. We identified that the demographics of urologic workforce is changing. There is also significant geographic variability regarding race/gender concordance between urologists and their patients. We found that female and URM providers are typically younger and finished residency later, both in the non-academic and academic urologist populations. In addition, the highest proportion of URM urologic practice in the South Atlantic section (37.9\%) and nearly half of academic URM urologists practice in the Mid-Atlantic (21.4\%) or East North-Central (15.6\%) sections. These geographic areas thereby become pockets of diversity within the workforce and can be targeted for future research on the impact of concordance on health metrics.

\section{Impact of racial/ethnic concordance on bealth outcomes}

There is evidence to support the impact of concordance on the perception of care by patients. A cohort study by 
Table 3 Demographic characteristics of academic urologists, stratified by URM status

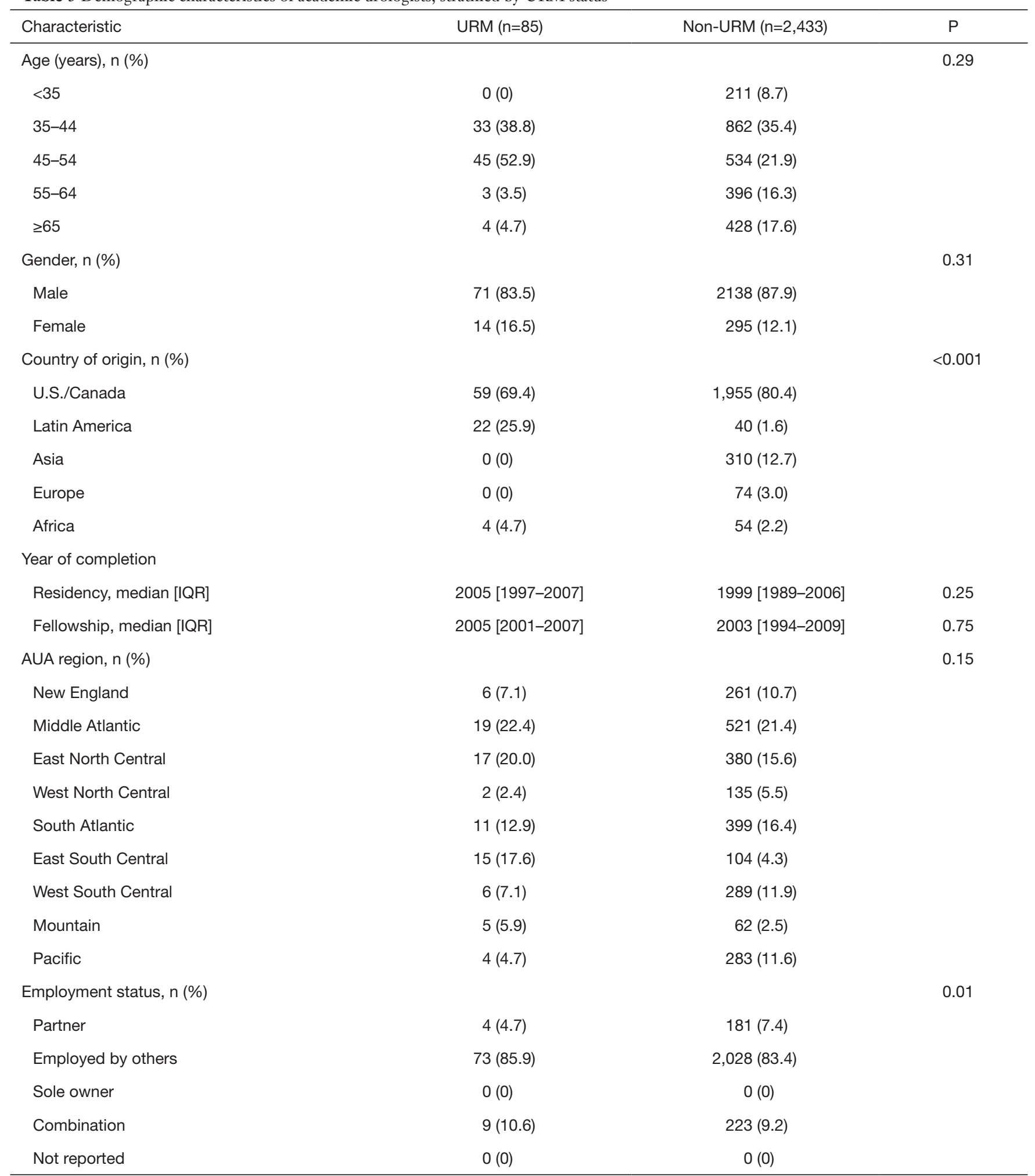

Counts reported are weighted and rounded to the nearest whole number. URM, underrepresented in medicine; AUA, American Urological Association. 

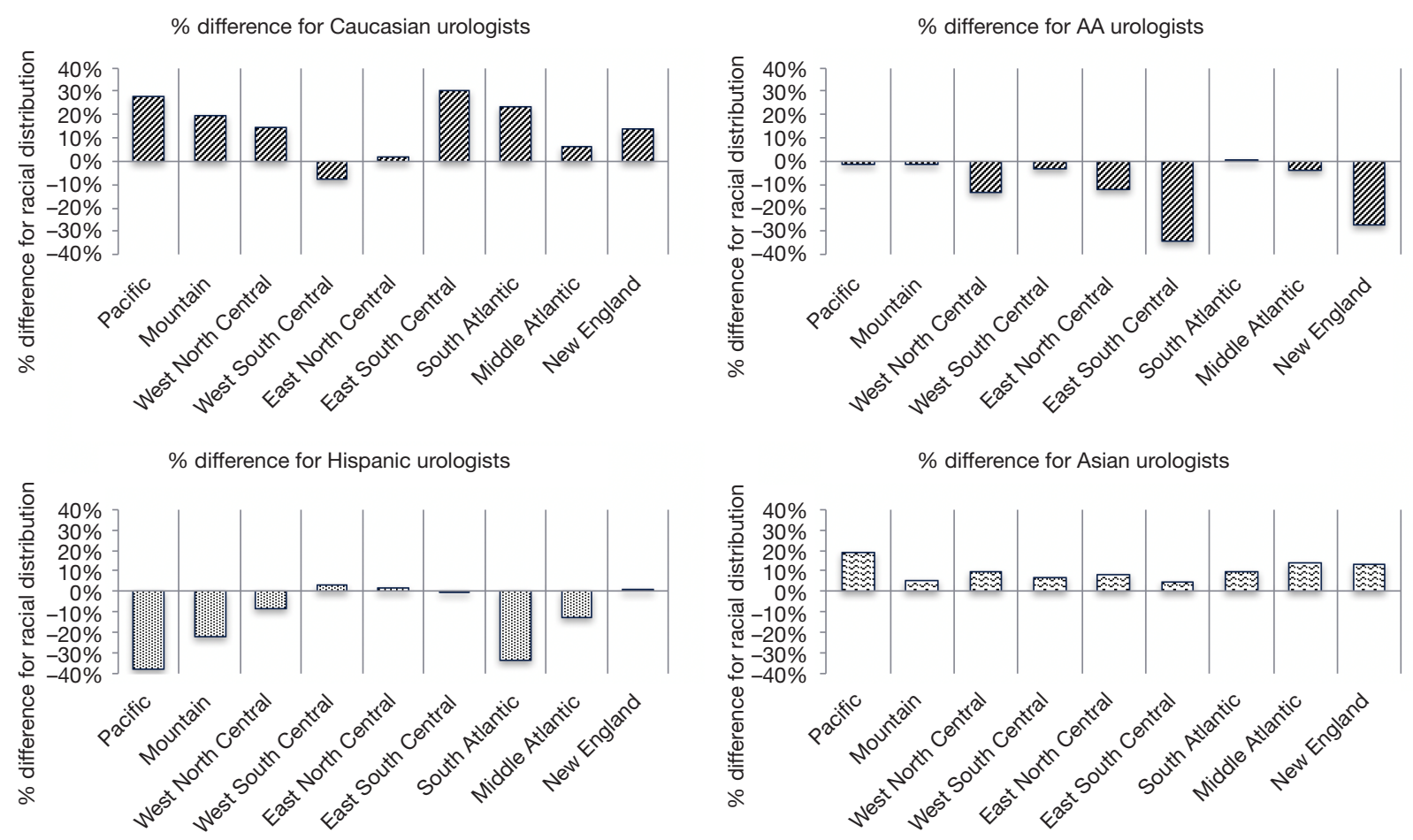

Figure 1 Comparison of racial/ethnic distributions between the general population and urologist population, stratified by AUA census region. AUA, American Urological Association.

Cooper et al. explored the effect of racial concordance in patient-physician communication in primary care encounters of 252 African-American (AA) and Caucasian patients (10). The authors found race-concordant visits to be longer with higher mean ratings of positive patient effect (the sum of ratings of engagement, interest, friendliness, and responsiveness) compared to race-discordant visits. Patient ratings of care were higher in race-concordant visits even when adjusting for communication behavior and specific race.

Others have found the impact of race/ethnicity concordance on patient satisfaction to be less clear (16). Blanchard et al. reported on patient satisfaction and perception of respect by patients using data from the Commonwealth Fund 2001 Quality of Care telephone survey. Patient-physician discordance was present in $14.4 \%$ of white patients, $52.2 \%$ of Asian patients, $69.3 \%$ of Hispanics, and $70.5 \%$ of AA patients. White and Asian patients were less likely to report being treated with disrespect in concordance relationships. However, surprisingly Hispanic patients were significantly more likely to report disrespect (OR, 2.42, 95\% CI: $1.23-4.73$ ) in a patient-provider concordance visit, even after controlling for sociodemographic factors. In addition, concordance between patients and social class was associated with lower rates of perceived disrespect. The authors believed cultural concordance, availability of hospital-related resources such as subspecialists and diagnostic procedures, and communication may have a stronger influence on satisfaction than race concordance alone.

A cross-sectional analysis of 7,070 adults in the 2010 Medical Expenditure Panel Survey (17) showed that nonwhite providers cared for a disproportionately large portion (53.5\%) of minority patients. Patients from underserved groups were more often seen by non-white providers, report fair or poor health status, and use emergency departments for care. The authors highlight the need for increased physician workforce diversity that offsets the disproportionate role of minority providers in underserved areas. They posit that improved minority recruitment at the medical school level is likely required.

\section{Impact of racial/ethnic diversity in academic medicine}

Diversity within academic educators may impact mentorship opportunities for students and junior faculties as well as the medical student's career choice $(18,19)$. Wright 
et al investigated the opinions of highly regarded physician mentors of a diverse group of medical learners at Johns Hopkins University School of Medicine (20). They found that learners generally prefer role models that are similar to them. Role modeling is also found to be easier for mentors when learners resemble the teachers. Their findings also suggest that URM physicians may be better role models for URM learners (20). Our study shows only $12 \%$ of URM providers work in academic medicine.

Mentorship is a critical aspect of academic achievement and mentorship success may be influenced by racial/ ethnic concordance. For URM faculty their own lack of mentorship may drive a sense of responsibility to those juniors which often conflicts with academic pursuits $(21,22)$. A study of URM Faculty at University of California, San Francisco explored the scarcity of racially concordant, available mentors in academic medicine (23). Mentorship correlated with career satisfaction and support. Yet mentorship remained fragmented due to the limited numbers of URM senior mentors $(23,24)$.

Our study has several limitations. Data is restricted to what is available in the U.S. and AUA Census data. For the U.S. Census, the derivation of weighted estimates of the general population from the 2010 Census introduces some degree of error. The AUA census data is also subject to sampling and estimate error due to the data being selfreported and survey-based. In addition, racial distribution of patients and urologists in a certain geographic location does not necessarily mean concordance visits and a more granular data collection is needed to provide a more accurate estimate. To our knowledge, this is the first ecological study of racial/ethnic concordance of practicing urologists and the U.S. population.

\section{Conclusions}

This study provides the first look at diversity within urology relative to the general population. We show that URM urologists tend to be younger with a higher proportion of female providers, indicating a gradual shift towards a more diverse field of urology. A more complete understanding of how diversity impacts patient care and satisfaction will significantly change how urologic care is provided in the increasingly diverse US.

\section{Acknowledgements}

None.

\section{Footnote}

Conflicts of Interest: The authors have no conflicts of interest to declare.

Ethical Statement: The study was approved for Exempt status by our institutional review board.

\section{References}

1. Thom DH, Ribisl KM, Stewart AL, et al. Further Validation and Reliability Testing of the Trust in Physician Scale. Med Care 1999;37:510-7.

2. Fiscella K, Meldrum S, Franks P, et al. Patient trust: Is it related to patient-centered behavior of primary care physicians? Med Care 2004;42:1049-55.

3. Safran DG, Taira DA, Rogers WH, et al. Linking primary care performance to outcomes of care. J Fam Pract 1998;47:213-20.

4. O'Malley AS, Sheppard VB, Schwartz M, et al. The role of trust in use of preventive services among low-income African-American women. Prev Med 2004;38:777-85.

5. Street RL, O'Malley KJ, Cooper LA, et al. Understanding Concordance in Patient- Physician Relationships : Personal and Ethnic. Ann Fam Med 2008;6:198-205.

6. Saha S, Komaromy M, Koepsell TD, et al. Patientphysician racial concordance and the perceived quality and use of health care. Arch Intern Med 1999;159:997-1004.

7. LaVeist TA, Nuru-Jeter A, Jones KE. The association of doctor-patient race concordance with health services utilization. J Public Health Policy 2003;24:312-23.

8. Laveist TA, Nuru-Jeter A. Is Doctor-Patient Race Concordance Associated with Greater Satisfaction with Care? J Health Soc Behav 2002;43:296.

9. King WD, Wong MD, Shapiro MF, et al. Does racial concordance between HIV-positive patients and their physicians affect the time to receipt of protease inhibitors? J Gen Intern Med 2004;19:1146-53.

10. Cooper LA, Roter DL, Johnson RL, et al. PatientCentered Communication, Ratings of Care, and Concordance of Patient and Physician Race. Ann Intern Med 2003;139:907-15.

11. Stevens GD, Mistry R, Zuckerman B, et al. The parentprovider relationship: Does race/ethnicity concordance or discordance influence parent reports of the receipt of high quality basic pediatric preventive services? J Urban Health 2005;82:560-74.

12. Weisse CS, Foster KK, Fisher EA. The influence 
of experimenter gender and race on pain reporting: Does racial or gender concordance matter? Pain Med 2005;6:80-7.

13. Kumar D, Schlundt DG, Wallston KA. Patient-physician race concordance and its relationship to perceived health outcomes. Ethn Dis 2009;19:345-51.

14. Cooper-Patrick L, Gallo JJ, Gonzales JJ, et al. Race, gender, and partnership in the patient-physician relationship. JAMA 1999;282:583-589.

15. Gordon HS, Street RL, Sharf BF, et al. Racial differences in doctors' information-giving and patients' participation. Cancer 2006;107:1313-20.

16. Blanchard J, Nayar S, Lurie N. Patient-provider and patient-staff racial concordance and perceptions of mistreatment in the health care setting. J Gen Intern Med 2007;22:1184-9.

17. Marrast LM, Zallman L, Woolhandler S, et al. Minority physicians' role in the care of underserved patients: Diversifying the physicianworkforce may be key in addressing health disparities. JAMA Intern Med 2014;174:289-91.

18. Brancati FL, Mead LA, Levine DM, et al. Early Predictors of Career Achievement in Academic Medicine. JAMA 1992;267:1372-6.

19. Saha S, Guiton G, Wimmers PF, et al. Student body racial and ethnic composition and diversity-related outcomes in US medical schools. JAMA 2008;300:1135-45.

20. Wright SM, Carrese JA. Serving as a physician role model for a diverse population of medical learners. Acad Med 2003;78:623-8.

21. Cochran A, Elder WB, Crandall M, et al. Barriers to advancement in academic surgery: Views of senior residents and early career faculty. Am J Surg 2013;206:661-6.

22. Pololi L, Cooper LA, Carr P. Race, disadvantage and faculty experiences in academic medicine. J Gen Intern Med 2010;25:1363-9.

23. Mahoney MR, Wilson E, Odom KL, et al. Minority faculty voices on diversity in academic medicine: Perspectives from one school. Acad Med 2008;83:781-6.

24. Odom KL, Roberts LM, Johnson RL, et al. Exploring obstacles to and opportunities for professional success among ethnic minority medical students. Acad Med 2007;82:146-53.
Cite this article as: Washington SL 3rd, Baradaran N, Gaither TW, Awad MA, Murphy GP, Downs TM, Breyer BN. Racial distribution of urology workforce in United States in comparison to general population. Transl Androl Urol 2018;7(4):526-534. doi: 10.21037/tau.2018.05.16 